\title{
Erratum: Existence and uniqueness of anti-periodic solutions for prescribed mean curvature Rayleigh equations
}

Jin $\mathrm{Li}^{1 *}$ and Zaihong Wang ${ }^{2}$

"Correspondence:

lijin7912@gmail.com

${ }^{1}$ School of Science, Jiujiang

University, Jiujiang, 332005, China

Full list of author information is

available at the end of the article

\begin{abstract}
In this paper, we give a complementary proof on the paper 'Existence and uniqueness of anti-periodic solutions for prescribed mean curvature Rayleigh equations'.
\end{abstract}

Keywords: complementary proof; prescribed mean curvature Rayleigh equations

\section{Introduction}

In [1], the authors were concerned with the existence and uniqueness of anti-periodic solutions of the following prescribed mean curvature Rayleigh equation:

$$
\left(\frac{x^{\prime}}{\sqrt{1+x^{\prime 2}}}\right)^{\prime}+f\left(t, x^{\prime}(t)\right)+g(t, x(t))=e(t)
$$

where $e \in C(R, R)$ is $T$-periodic, and $f, g \in C(R \times R, R)$ are $T$-periodic in the first argument, $T$ is a constant.

The paper mentioned above obtained the main result by using Mawhin's continuation theorem in the coincidence degree theory. Unfortunately, the proof of main result Theorem 3.1 (see [1]) has a serious problem: $F_{\mu}(x)=\mu L\left(Q_{1}\left(t, x_{1}, x_{2}\right)\right)$ where $Q_{1}$ depends on $\psi\left(x_{2}\right)$ and $\psi(x)=\frac{x}{\sqrt{1-x^{2}}}$ which is only defined for $|x|<1$ and cannot be continuously extended; therefore, $F_{\mu}$ should not be defined on $\bar{\Omega}=\{x \in X:\|x\|<M\}$ since $\left|x_{2}(t)\right|>1$ can occur, where $\|x\|=\max \left\{\left\|x_{1}\right\|_{\infty},\left\|x_{2}\right\|_{\infty}\right\}$ and $M=1+\max \left\{D_{1}, D_{2}\right\}$.

In this paper, we shall give a complementary proof to correct the errors.

\section{Complementary proof}

Rewrite (1.1) in the equivalent form as follows:

$$
\left\{\begin{array}{l}
x_{1}^{\prime}(t)=\psi\left(x_{2}(t)\right)=\frac{x_{2}(t)}{\sqrt{1-x_{2}^{2}(t)}}, \\
x_{2}^{\prime}(t)=-f\left(t, \psi\left(x_{2}(t)\right)\right)-g\left(t, x_{1}(t)\right)+e(t),
\end{array}\right.
$$

(C) $2014 \mathrm{Li}$ and Wang: licensee Springer. This is an Open Access article distributed under the terms of the Creative Commons Attribution License (http://creativecommons.org/licenses/by/4.0), which permits unrestricted use, distribution, and reproduction in any medium, provided the original work is properly credited. 
where $\psi(x)=\frac{x}{\sqrt{1-x^{2}}}$. In [1], the authors embed (2.1) into a family of equations with one parameter $\lambda \in(0,1]$,

$$
\left\{\begin{array}{l}
x_{1}^{\prime}(t)=\lambda \frac{x_{2}(t)}{\sqrt{1-x_{2}^{2}(t)}}=\lambda \psi\left(x_{2}(t)\right), \\
x_{2}^{\prime}(t)=-\lambda f\left(t, \psi\left(x_{2}(t)\right)\right)-\lambda g\left(t, x_{1}(t)\right)+\lambda e(t) .
\end{array}\right.
$$

They have proved that there exists a constant $D_{1}>0$ such that

$$
\left|x_{1}^{\prime}\right|_{2} \leq D_{1}, \quad \text { and } \quad\left|x_{1}\right|_{\infty} \leq D_{1}
$$

and there exists $\eta \in[0, T]$ such that $x_{2}(\eta)=0$.

In fact, to use the continuation theorem, it suffices to prove that there exists a positive constant $0<\varepsilon_{0} \ll 1$ such that, for any possible solution $\left(x_{1}(t), x_{2}(t)\right)$ of $(2.2)$, the following condition holds:

$$
\left|x_{2}(t)\right|<1-\varepsilon_{0}
$$

In what follows, we shall give a complementary proof for the main result in [1] by giving a proof of (2.4).

In [1], the authors assume that

$\left(\mathrm{H}_{1}\right)\left(g\left(t, x_{1}\right)-g\left(t, x_{2}\right)\right)\left(x_{1}-x_{2}\right)<0$, for all $t, x_{1}, x_{2} \in R$ and $x_{1} \neq x_{2}$;

$\left(\mathrm{H}_{2}\right)$ there exists $l>0$ such that

$$
\left|g\left(t, x_{1}\right)-g\left(t, x_{2}\right)\right| \leq l\left|x_{1}-x_{2}\right| \quad \text { for all } t, x_{1}, x_{2} \in R
$$

$\left(\mathrm{H}_{3}\right)$ there exists $\beta, \gamma$ such that

$$
\gamma \leq \liminf _{|x| \rightarrow \infty} \frac{f(t, x)}{x} \leq \limsup _{|x| \rightarrow \infty} \frac{f(t, x)}{x} \leq \beta, \quad \text { uniformly in } t \in R
$$

$\left(\mathrm{H}_{4}\right)$ for all $t, x \in R$,

$$
f\left(t+\frac{T}{2},-x\right)=-f(t, x), \quad g\left(t+\frac{T}{2},-x\right)=-g(t, x), \quad e\left(t+\frac{T}{2}\right)=-e(t) .
$$

Under the conditions mentioned above, we prove that (2.4) holds.

Since $\left|x_{1}\right|_{\infty}<D_{1}$ and $g, e$ are continuous, we find that there exists $M_{3}>0$ such that

$$
-M_{3}<-g\left(t, x_{1}(t)\right)+e(t)<M_{3}, \quad \forall t \in R .
$$

By $\left(\mathrm{H}_{3}\right)$, there exists a positive constant $M_{4}>0$ such that

$$
f(t, x) \geq \gamma x-M_{4}, \quad \forall x>0 \text { and } \forall t \in R .
$$

Next, we shall prove that

$$
x(t) \leq \frac{M_{3}+M_{4}}{\sqrt{\left(M_{3}+M_{4}\right)^{2}+\gamma^{2}}}, \quad \forall t \in R .
$$


Assume by contradiction that there exist $t_{2}^{*}>t_{1}^{*}>\eta$ such that

$$
x_{2}\left(t_{1}^{*}\right)=\frac{M_{3}+M_{4}}{\sqrt{\left(M_{3}+M_{4}\right)^{2}+\gamma^{2}}}, \quad x_{2}\left(t_{2}^{*}\right)>\frac{M_{3}+M_{4}}{\sqrt{\left(M_{3}+M_{4}\right)^{2}+\gamma^{2}}},
$$

and

$$
x_{2}(t)>\frac{M_{3}+M_{4}}{\sqrt{\left(M_{3}+M_{4}\right)^{2}+\gamma^{2}}}, \quad \text { for } t \in\left(t_{1}^{*}, t_{2}^{*}\right) \text {. }
$$

Noticing that $\lambda \in(0,1]$, we have, $\forall t \in\left(t_{1}^{*}, t_{2}^{*}\right)$,

$$
x_{2}^{\prime}(t)=\lambda\left(-f\left(t, \psi\left(x_{2}(t)\right)\right)-g\left(t, x_{1}(t)\right)+e(t)\right)<0,
$$

which is a contradiction.

By $\left(\mathrm{H}_{3}\right)$, there exists a positive constant $M_{5}>0$ such that

$$
f(t, x) \leq \beta x+M_{5}, \quad \forall x<0 \text { and } \forall t \in R .
$$

By using a similar argument, we can prove that

$$
x_{2}(t) \geq-\frac{M_{3}+M_{5}}{\sqrt{\left(M_{3}+M_{5}\right)^{2}+\beta^{2}}}, \quad \text { for } t \in R \text {. }
$$

Therefore, we get from the continuity of $x_{2}(t)$, for any solution $\left(x_{1}(t), x_{2}(t)\right)$ of $(2.2)$,

$$
-\frac{M_{3}+M_{5}}{\sqrt{\left(M_{3}+M_{5}\right)^{2}+\beta^{2}}} \leq x_{2}(t) \leq \frac{M_{3}+M_{4}}{\sqrt{\left(M_{3}+M_{4}\right)^{2}+\gamma^{2}}}, \quad \forall t \in R .
$$

Consequently, (2.4) holds.

Putting

$$
\Omega=\left\{x=(x, x) \in C_{T}^{0, \frac{1}{2}}\left(R, R^{2}\right)=X:\|x\|<M,\left|x_{2}(t)\right|<1-\varepsilon_{0}\right\},
$$

we can use Mawhin's continuation theorem on $\Omega$.

Competing interests

The authors declare that they have no competing interests.

Authors' contributions

All authors contributed equally to the writing of this paper. All authors read and approved the final manuscript.

\section{Author details}

${ }^{1}$ School of Science, Jiujiang University, Jiujiang, 332005, China. ${ }^{2}$ School of Mathematical Sciences, Capital Normal University, Beijing, 100048, China.

\section{Acknowledgements}

The authors would like to thank Professor J Webb for pointing out the errors of the paper [1].

Received: 18 August 2014 Accepted: 18 August 2014 Published online: 25 September 2014 
References

1. Li, J, Wang, Z: Existence and uniqueness of anti-periodic solutions for prescribed mean curvature Rayleigh equations. Bound. Value Probl. 2012, 109 (2012)

doi:10.1186/s13661-014-0204-5

Cite this article as: Li and Wang: Erratum: Existence and uniqueness of anti-periodic solutions for prescribed mean curvature Rayleigh equations. Boundary Value Problems 2014 2014:204.

Submit your manuscript to a SpringerOpen ${ }^{\circ}$ journal and benefit from:

- Convenient online submission

- Rigorous peer review

- Immediate publication on acceptance

Open access: articles freely available online

- High visibility within the field

- Retaining the copyright to your article

Submit your next manuscript at $>$ springeropen.com 